\title{
Syncope and profound bradycardia associated with intrauterine contraceptive procedures
}

\author{
Aisling Baird, ${ }^{1}$ Jane Dickson, ${ }^{2}$ Mary Jensen, ${ }^{3}$ Martin Talbot ${ }^{4}$
}

\author{
${ }^{1}$ Consultant in Sexual and \\ Reproductive Health, Liverpool \\ Community Health, Central \\ Abacus, Liverpool, UK \\ ${ }^{2}$ Community Specialist, \\ Contraception and Sexual \\ Health, Greenwich Community \\ Health Services, Oxleas NHS \\ Foundation Trust, London, UK \\ ${ }^{3}$ Associate Specialist in Sexual \\ and Reproductive Health, \\ Centre for Contraception and \\ Sexual Health, Victoria Health \\ Centre, Glasshouse Street, \\ Nottingham, UK \\ ${ }^{4}$ Consultant Physician in \\ Genitourinary Medicine/HIV, \\ Department of Genitourinary \\ Medicine, Sheffield Teaching \\ Hospitals NHS Foundation Trust, \\ Royal Hallamshire Hospital, \\ Sheffield, UK
}

\section{Correspondence to}

Dr Aisling Baird, Liverpool Community Health, Central

Abacus, Citrus House, 40-46 Dale Street, Liverpool L2 5SF, UK; aisling.baird@liverpoolch.nhs.uk

Received 28 August 2011 Accepted 22 December 2011 Published Online First

17 January 2012

\begin{abstract}
There has been recent interest in this Journal concerning the occurrence of profound bradycardia with impaired consciousness during insertion of intrauterine contraceptive devices or systems. Questions have been raised regarding the requirement for medication for reversal of the condition, the role of the nurse practitioner in the light of this, and the effects upon sexual and reproductive health care service delivery. We present three cases where this condition affected patients under our care and suggest that although very infrequent, it is important. Medication for treatment and staff trained to administer it should always be available.
\end{abstract}

\section{Introduction}

Bradycardia and syncope during insertion of an intrauterine contraceptive device (IUD) or system (IUS) are recognised complications and are thought to be due to stimulation of the vagus nerve. Although this is not a common occurrence, women undergoing the procedure may rapidly become profoundly unwell. The guidance on intrauterine contraception of the UK Faculty of Sexual and Reproductive Healthcare (FSRH) states that the availability of appropriate emergency medication, including atropine, during IUD/IUS insertion is essential, and this is a service standard for resuscitation in sexual health services. ${ }^{12}$

In a retrospective chart review of 545 patients seen over a 3 -year period, Farmer and Webb reported that bradycardia [pulse $<60$ beats per minute (bpm)] occurred in $1.8 \%$ of IUD insertions. ${ }^{3}$ They mentioned earlier ECG studies that had estimated the occurrence of bradycardia as $32 \%$, although this was in an era of more inflexible, rigid devices, specifically the Dalkon Shield, and referred to other studies reporting syncope in $2.1 \%$ of insertions. In recent correspondence in this Journal, Gormley and Eady drew attention to the rarity of the condition and called for evidence that a doctor must be present for all insertions, yet offered only anecdotal evidence as to the lack of need for atropine. ${ }^{4}$ Hollingworth used personal experience and that of her colleagues to query the FSRH guidelines on resuscitation in sexual health services and specifically the availability of atropine. ${ }^{5}$ Mansour conducted a poll of over 70 inserters, none of whom had ever needed to use atropine - the implication being that simple first aid measures should be sufficient to reverse the condition. ${ }^{6}$ Mehigan believed the FSRH guidance to be "unrealistic". Gormley and Eady championed the preparedness of the woman and the universal use of local anaesthesia; however, there is little evidence to support the assumption that pain is part of the pathogenesis of this condition.

Dickson et al. responded to these communications in 2011, setting out the origin of the Faculty standards. ${ }^{8}$ Specifically, and pertinent to our communication, they discussed the Resuscitation Council guideline that intravenous atropine should be administered if adverse signs such as shock or syncope are present. We now report three cases of the condition in patients under our care, in whom conservative measures were ineffective but who responded successfully to the administration of atropine.

\section{Case histories}

\section{Case 1}

This 35-year-old woman, para 3, presented for IUD insertion. She was well and had no medical or gynaecological problems. On the morning of the procedure she had not had breakfast. Preliminary examination was normal. An IUD was inserted without difficulty, with no analgesia. Immediately following insertion, she became pale, sweaty, vacant and unresponsive [Glasgow Coma Scale (GCS) 10]. Her pulse was found to be $30 \mathrm{bpm}$ and thready. She was laid flat and her legs elevated. This produced no response. Atropine sulphate $600 \mu \mathrm{g}$ was administered intravenously, 
following which she became responsive (GCS 15) and her pulse rose to $60 \mathrm{bpm}$.

\section{Case 2}

This 46-year-old woman, para 3, was referred to a specialist clinic for replacement of her IUS. She reported symptoms suggestive of the menopause with hot flushes and difficulty sleeping. Her IUS had been in situ for 6 years following a "difficult fitting". When questioned about the difficulty she simply said that she had not been very well afterwards. Preliminary examination was normal. Her pre-procedure pulse was $60 \mathrm{bpm}$ and blood pressure (BP) was $104 / 70 \mathrm{mmHg}$. The internal os was stenosed but the fundus was sounded successfully and the cavity measured $7 \mathrm{~cm}$. The IUS 'snagged' at the internal os on traction, but was then removed easily.

At this point the patient had a vasovagal episode and the replacement procedure was abandoned. She initially recovered spontaneously and was able to communicate. Her pulse fluctuated between 42 and $48 \mathrm{bpm}$. Her BP was normal. Simple first aid measures were continued. At one point her pulse returned to $60 \mathrm{bpm}$. Her head was raised for her to have a sip of water, at which point she collapsed again, her pulse falling to $36 \mathrm{bpm}$. Oxygen therapy was commenced and $200 \mu \mathrm{g}$ atropine sulphate was administered intravenously. She was now completely unresponsive, GCS 3, her pulse remained $36 \mathrm{bpm}$ and the heart sounds were very faint. There had been no response to the initial small dose of atropine. After 10 minutes another $200 \mu \mathrm{g}$ atropine was administered, following which her condition improved, GCS 14 and pulse $60 \mathrm{bpm}$. She was taken to hospital where she only required observation for a short time.

\section{Case 3}

This 40-year-old nulliparous woman attended for IUS insertion. Preliminary assessment was unremarkable. Judd-Allis forceps were applied to the anterior cervical lip, following which she commented that she felt unwell. A flexible sound was introduced into the uterus, at which point she became pale, lost consciousness (GCS 4) and had an anoxic seizure. The patient was placed into the recovery position and oxygen administered. Her pulse was weak and slow (45 bpm). Although the seizure ceased, she remained unconscious and there was no sign of recovery, at which point $600 \mu \mathrm{g}$ atropine was administered intravenously. The patient began to gain consciousness immediately and after 1 minute her pulse increased to $72 \mathrm{bpm}$. She recovered fully within 30 minutes and was allowed home.

\section{Discussion}

Atropine is an alkaloid from the plant Atropa belladonna and is a competitive antagonist to acetylcholine. It blocks muscarinic receptors in the autonomic nervous system, thus counteracting the effects of vagal stimulation. In healthy individuals this results in a modest tachycardia, since it is the parasympathetic nervous system that is blocked, rather than the sympathetic stimulated. In therapeutics it is a relatively safe and straightforward drug, which is why several doses may be administered.

The Resuscitation Council (UK) lists shock and syncope as "adverse features" of bradycardia, for which atropine should be administered. It may be given in repeated doses to a maximum of $3 \mathrm{mg} .{ }^{9}$ Oxygen should also be administered.

A GCS of 3, as exhibited by Case 2, indicates severe unconsciousness and is the lowest possible score: even a score of 10 (Case 1) is classified as moderate impairment. It could be argued that given time, Case 1 might have responded to simple first aid measures, yet all three patients, by definition, showed clinical features indicating cerebral hypoperfusion and hypoxia. This was particularly severe in Case 3, who developed anoxic seizures.

It could also be argued that insertion might have been deferred in Case 1 due her not having eaten that morning, but there is no convincing evidence that there are any definite 'red flag' features in a woman that would alert one to an increased possibility of a vasovagal episode. There is no evidence that hunger contributes to this, nor is there evidence that pain relief is preventive. Being welcoming, calm and friendly and providing adequate analgesia ${ }^{3}$ are all appropriate measures for women and are good professional practice, but do not solve the problem of vagal stimulation.

Vasovagal episodes associated with cervical instrumentation or dilatation, sometimes referred to as 'cervical shock', may be rare, but the collapse may be profound, as we saw in two of our patients. In the other (Case 1) the degree of bradycardia was remarkable. The second patient had only a modest dose of atropine initially and this case illustrates that conservative first aid measures, or an inadequate dose of atropine, may be ineffective. We would be unhappy to undertake IUD insertion without the availability of suitable resuscitation equipment. In particular we advocate the availability of atropine for intravenous use and the appropriate training of staff to be able to administer it.

Competing interests Dr Jane Dickson is the current ViceChair of the FSRH Clinical Standards Committee.

Provenance and peer review Not commissioned; externally peer reviewed.

\section{References \\ 1 Faculty of Sexual \& Reproductive Healthcare Clinical Effectiveness Unit. Intrauterine Contraception. November 2007. http://www.fsrh.org/pdfs/ CEUGuidanceIntrauterineContraceptionNov07.pdf [accessed 11 October 2011]. \\ 2 Faculty of Sexual \& Reproductive Healthcare. Service Standards for Resuscitation. October 2010. http://www.fsrh.org/pdfs/ ServiceStandardsResuscitation.pdf [accessed 11 October 2011]. \\ 3 Farmer M, Webb A. Intrauterine device insertion-related complications: can they be predicted? J Fam Plann Reprod Health Care 2003;29:227-231.}


4 Gormley M, Eady A. IUD fitters and training in resuscitation [Letter]. J Fam Plann Reprod Health Care 2010;36:254.

5 Hollingworth B. Need for IUD fitters to have expertise in resuscitation [Letter]. J Fam Plann Reprod Health Care 2010;36:179-180.

6 Mansour D. Nurse training and the need for IUD fitters to have expertise in resuscitation [Letter]. J Fam Plann Reprod Health Care 2010;36:180; author reply 180.
7 Mehigan S. Reply to Hollingworth and Mansour [Letter]. J Fam Plann Reprod Healthcare 2010:36;180.

8 Dickson J, Kasliwal A, Randall S. IUD/IUS insertions and atropine [Letter]. J Fam Plann Reprod Health Care 2011;37:118-119.

9 Resuscitation Council (UK). 2010 Resuscitation Guidelines. Adult bradycardia algorithm. http://www.resus.org.uk/pages/ bradalgo.pdf [accessed 16 August 2011]. 